\title{
Thermodynamic parameters at kinetic freeze-out in relativistic heavy-ion collisions using Tsallis statistics
}

\author{
Rajendra Nath Patra, ${ }^{a, *}$ Bedangadas Mohanty ${ }^{b}$ and Tapan K. Nayak ${ }^{b, c}$ \\ ${ }^{a}$ University of Jammu, Jammu 180006, India \\ ${ }^{b}$ National Institute of Science Education and Research, Jatni 752050, India \\ ${ }^{c}$ CERN, CH 1211, Geneva 23, Switzerland \\ E-mail: rajendra.nath.patra@cern.ch, rajendrapatra07@gmail.com
}

\begin{abstract}
Thermodynamic properties of the hot and dense system produced in relativistic heavy-ion collisions have been studied by analyzing the transverse momentum $\left(p_{\mathrm{T}}\right)$ spectra of emitted particles (pions, kaons, and protons). The spectra are fitted using the non-extensive Tsallis statistics. The fit parameters, $q$ and $T$, provide the degree of deviation of the system from an equilibrium state and the effective temperature at freeze-out, respectively. These parameters are presented as a function of collision energy, collision centrality, and different fit ranges in $p_{\mathrm{T}}$ for available experimental data at Relativistic Heavy Ion Collider (RHIC) and the Large Hadron Collider (LHC) energies. An anti-correlation between the two fit parameters has been observed. With the increase of the collision energy, $q$ increases in a systematic manner whereas $T$ has a decreasing trend. For central collisions, $q$ and $T$ have a strong dependence on the fitting ranges of $p_{\mathrm{T}}$, however, for peripheral collisions there is a minor dependency on $p_{\mathrm{T}}$. The Tsallis parameters are found to have mass ordering, which needs further investigation with the inclusion of radial flow.
\end{abstract}

\footnotetext{
*** The European Physical Society Conference on High Energy Physics (EPS-HEP2021), ***

*** 26-30 July $2021 * * *$

*** Online conference, jointly organized by Universität Hamburg and the research center DESY ***
}

\footnotetext{
*Speaker
} 


\section{Introduction}

Relativistic heavy-ion collisions, probed at experimental facilities of RHIC at Brookhaven National Laboratory and LHC at CERN, produce matter at extreme conditions of temperatures and energy densities in the form of quark-gluon plasma (QGP). The QGP is a transient stage which cools down rapidly $(7-10 \mathrm{fm} / \mathrm{c})$ and finally freezing in the form of outgoing hadrons. The space-time evolution of the system formed passes through two symbolic freeze-outs, chemical freeze-out and kinetic freeze-out. The colliding medium first reaches chemical equilibrium and then cools down by expansion. Due to this expansion of the system, the inelastic collisions cease when the mean free path for the interactions becomes comparable to the system size. This is known as the chemical freeze-out, at which point the abundances of different particle species become constant. After this stage, even if the relative fractions of the particles are constant, these particles continue to interact till a point where the final state interactions between the hadrons are no longer effective. After this kinetic freeze-out all the interactions cease and the (transverse) momentum spectra of the particles remain unchanged. The studies of the $p_{\mathrm{T}}$ spectra of the outgoing particles provide direct insight into the thermodynamical properties at kinetic freeze-out conditions. Boltzmann-Gibbs (BG) distribution function with the blast-wave model is largely used by many experiments [1-6] to explain the $p_{\mathrm{T}}$ spectra and hence calculate temperature at the kinetic freeze-out. However, the drawback of the BG blast-wave model is that it can explain $p_{\mathrm{T}}$ spectra only at low $p_{\mathrm{T}}$ ranges $(2-3 \mathrm{GeV} / c)[5-7]$.

The non-extensive Tsallis statistics is found to provide a better description of the $p_{\mathrm{T}}$ spectra at high-energy collisions [8-12], as it is successful in explaining the spectra up to high $p_{\mathrm{T}}$ ranges successfully. Tsallis statistics has been found to be appropriate to explain the $p_{\mathrm{T}}$ spectra in protonproton $(p p)[10,11]$ as well as in heavy-ion collisions [12]. Since this distribution function does not account for radial flow contributions, the temperatures obtained from the Tsallis fits are the effective temperatures, different from the kinetic freeze-out temperatures.

The invariant momentum distribution in terms of Tsallis distribution can be written as,

$$
E \frac{d^{3} N}{d p^{3}}=\frac{g V}{(2 \pi)^{3}} E\left(1+(q-1) \frac{E-\mu}{T}\right)^{-\frac{q}{q-1}}
$$

where $E, T$, and $\mu$ are energy, effective temperature, and chemical potential, respectively. $q$ is the entropy index that measures the deviation from the equilibrium of the system. Equation 1 in the limit $q \rightarrow 1$ represents statistics of equilibrium state i.e. Boltzmann-Gibbs statistics. Equation 1 can be written as,

$$
\frac{1}{2 \pi p_{\mathrm{T}}} \frac{d^{2} N}{d p_{\mathrm{T}} d y}=\frac{g V}{(2 \pi)^{3}} m_{\mathrm{T}} \cosh (y)\left(1+(q-1) \frac{m_{\mathrm{T}} \cosh (y)-\mu}{T}\right)^{-\frac{q}{q-1}} .
$$

Fitting the experimentally measured $p_{\mathrm{T}}$ spectra in terms of transverse mass $\left(m_{\mathrm{T}}\right)$ and rapidity $(y)$, one obtains the Tsallis parameters, $q$ and $T$. For all charged particles, at mid-rapidity, the above expression becomes,

$$
\frac{d^{2} N_{\mathrm{ch}}}{d p_{\mathrm{T}} d \eta}=2 p_{\mathrm{T}}^{2} \frac{V}{(2 \pi)^{2}} \sum_{i=1}^{3} g_{i}\left(1+(q-1) \frac{m_{\mathrm{T}, \mathrm{i}}-\mu}{T}\right)^{-\frac{q}{q-1}},
$$


where $N_{\text {ch }}$ is charged particle multiplicity and $\eta$ is the pseudo-rapidity. The sum runs over three most abundant particles, $\pi, \mathrm{K}$ and $\mathrm{p}$. Factor 2 in Equation 3 accounts for both particles and their anti-particles. The degeneracy factors $(g)$ are unity for pions and kaons and 2 for protons.

\section{Data analysis, results and discussion}

The thermodynamic properties at the freeze-out of the medium formed can be understood by analyzing the $p_{\mathrm{T}}$ spectra of hadrons at different collisions energies $\left(\sqrt{s_{\mathrm{NN}}}\right)$ and collision centralities. We have analyzed the $p_{\mathrm{T}}$ spectra of all charged particles and identified particles from STAR (Au-Au at $\sqrt{s_{\mathrm{NN}}}=7.7,11.5,14.519 .6,27,39,62.4$, and $\left.200 \mathrm{GeV}[3,4,13,14]\right)$ and ALICE (Pb-Pb at $\sqrt{s_{\mathrm{NN}}}=2.76$ and $5.02 \mathrm{TeV}[6,15-17]$ and $\mathrm{Xe}-\mathrm{Xe}$ at $\left.5.44 \mathrm{TeV}[18,19]\right)$ using Tsallis fits. Figure 1 shows the Tsallis fit to $p_{\mathrm{T}}$ spectra of pions for Xe-Xe collisions at $\sqrt{s_{\mathrm{NN}}}=5.44 \mathrm{TeV}$, fitted up to $p_{\mathrm{T}}$ range of $2 \mathrm{GeV} / \mathrm{c}$ (left) and $5 \mathrm{GeV} / \mathrm{c}$ (right) for different centrality classes. The quality of the fits can be inferred from the ratio plot shown in the lower panels. Similar fits are made for different collision systems and energies, and the results are presented in Ref. [20].
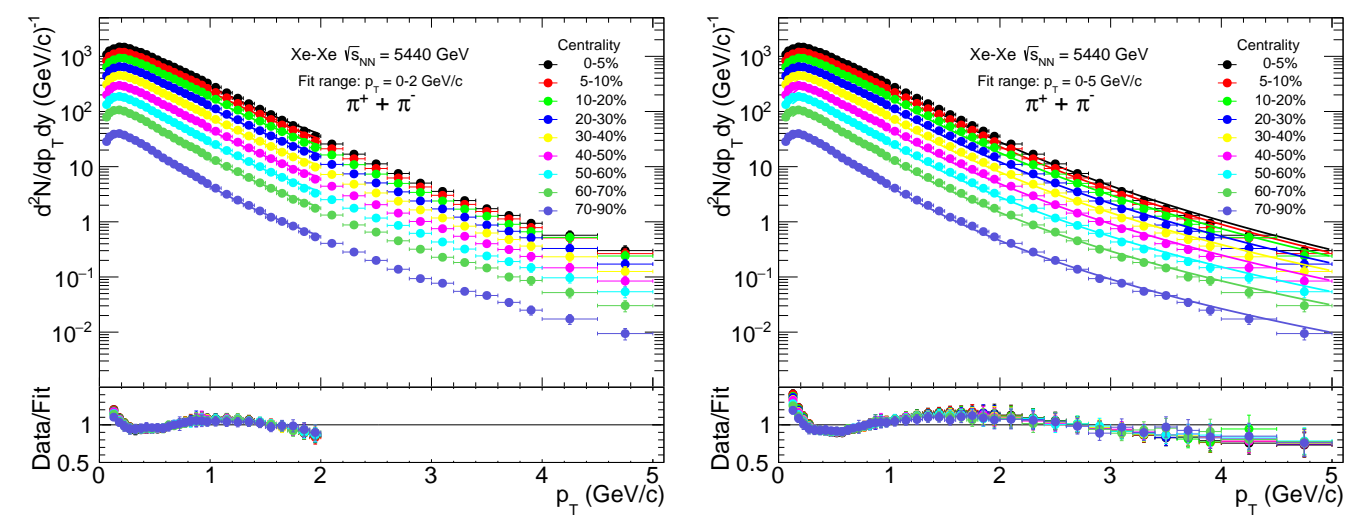

Figure 1: $p_{\mathrm{T}}$ spectra of pions for different centralities in Xe-Xe at $\sqrt{s_{\mathrm{NN}}}=5.44 \mathrm{TeV}$ [19], fitted using Tsallis distribution for different $p_{\mathrm{T}}$ ranges. The lower panels show the ratios of data to the obtained fits.

The variation of $q$ and $T$ as a function of collisions energy for central and peripheral collisions for pions are shown in Figure 2 for fits up to $2 \mathrm{GeV} / \mathrm{c}$ in $p_{\mathrm{T}}$. For both centralities, $q$ increases with $\sqrt{s_{\mathrm{NN}}}$ whereas $T$ decreases consistently. It can be seen that for all $\sqrt{s_{\mathrm{NN}}}, T$ is higher for central collisions compared to the peripheral ones. The variation of the parameters $q$ and $T$ with $\sqrt{s_{\mathrm{NN}}}$ for the identified pions, kaons, and protons are shown in Figure 3 . Here fits are made for $p_{\mathrm{T}}$ ranges of $2 \mathrm{GeV} / \mathrm{c}, 3 \mathrm{GeV} / \mathrm{c}$ and $5 \mathrm{GeV} / \mathrm{c}$ for pions, kaons and protons, respectively. We see that the Tsallis parameters have a mass dependency. For pions and kaons, $q$ increases with $\sqrt{s_{\mathrm{NN}}}$, but protons show an unusually different trend.

Tsallis parameters are found to have a dependency on the fitting range of $p_{\mathrm{T}}$ as presented in Figure 4. It can be seen that with the increase of the $p_{\mathrm{T}}$ range, $q$ decreases and then becomes almost constant after $p_{\mathrm{T}} \sim 6 \mathrm{GeV} / \mathrm{c}$. The variation of $T$ with increasing the $p_{\mathrm{T}}$ is also quite significant and at mid $p_{\mathrm{T}}(\sim 5-10 \mathrm{GeV} / \mathrm{c})$ the value is almost constant and then decreases. The mid- $p_{\mathrm{T}}$ region might be useful for different physics inferences as $q$ and $T$ almost remain constant. 

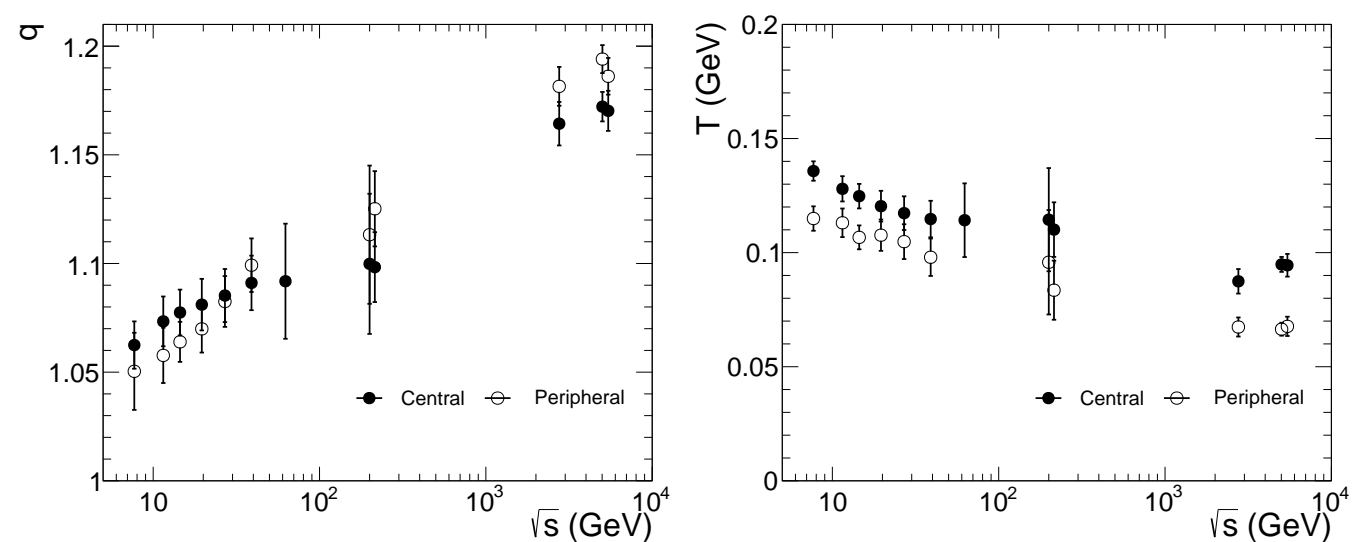

Figure 2: Tsallis parameters $q, T$ as a function of collisions energy at central and peripheral collisions. The parameters correspond to fitting of the $p_{\mathrm{T}}$ spectra up to $2 \mathrm{GeV} / \mathrm{c}$ for pions.
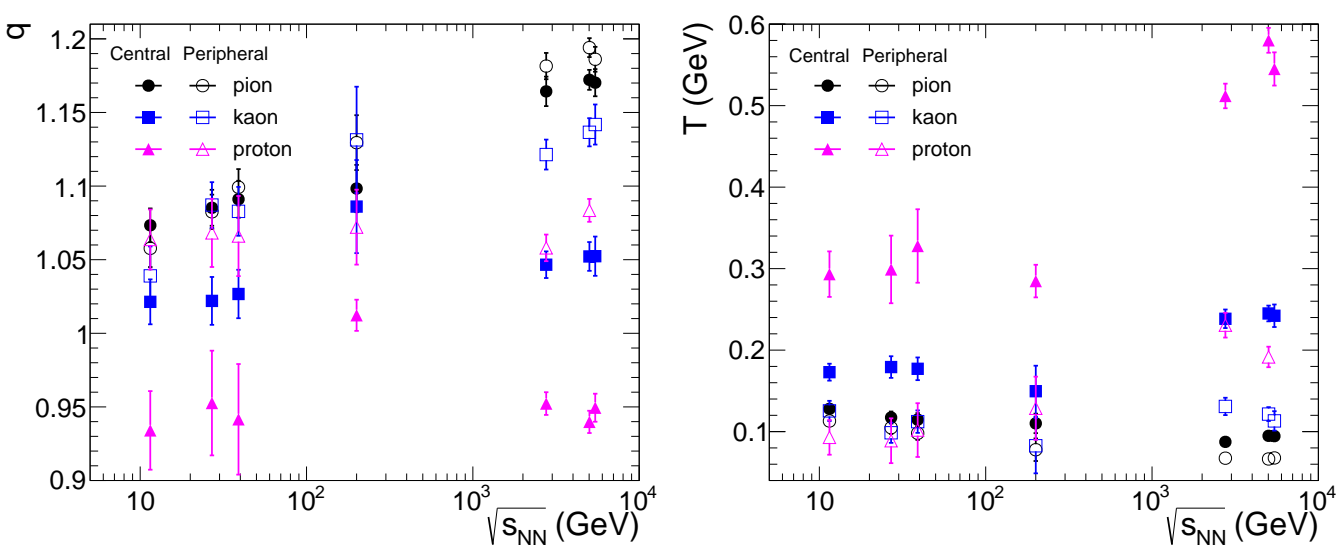

Figure 3: Tsallis parameters $q, T$ for pions, kaons and protons for fits up to $p_{\mathrm{T}}$ range of $2 \mathrm{GeV} / \mathrm{c}, 3 \mathrm{GeV} / \mathrm{c}$ and $5 \mathrm{GeV} / \mathrm{c}$, respectively are shown as a function of collision energy.

\section{Summary}

The Tsallis distribution function has been used to successfully fit the $p_{\mathrm{T}}$ spectra of charged particles and pions up to a large $p_{\mathrm{T}}$. The parameter $q$ shows an increasing trend with $\sqrt{s_{\mathrm{NN}}}$ where $T$ has a decreasing trend. In a centrality dependency study temperature $T$ has a consistently lower value in peripheral collisions compared to central collisions for all $\sqrt{s_{\mathrm{NN}}}$. The Tsallis parameters show a mass ordering dependency which is a consequence of the presence of radial flow effect. It is found that both $q$ and $T$ depend on $p_{\mathrm{T}}$ fitting ranges and the dependency is prominent in central collisions. The Tsallis parameters are almost constant at mid- $p_{\mathrm{T}}$ and independent of the $p_{\mathrm{T}}$ ranges.

\section{References}

[1] E. Schnedermann, J. Sollfrank and U. W. Heinz, Phys. Rev. C 48, 2462 (1993).

[2] B. Abelev et al., (STAR Collaboration), Phys. Rev. C 79, 034909 (2009). 

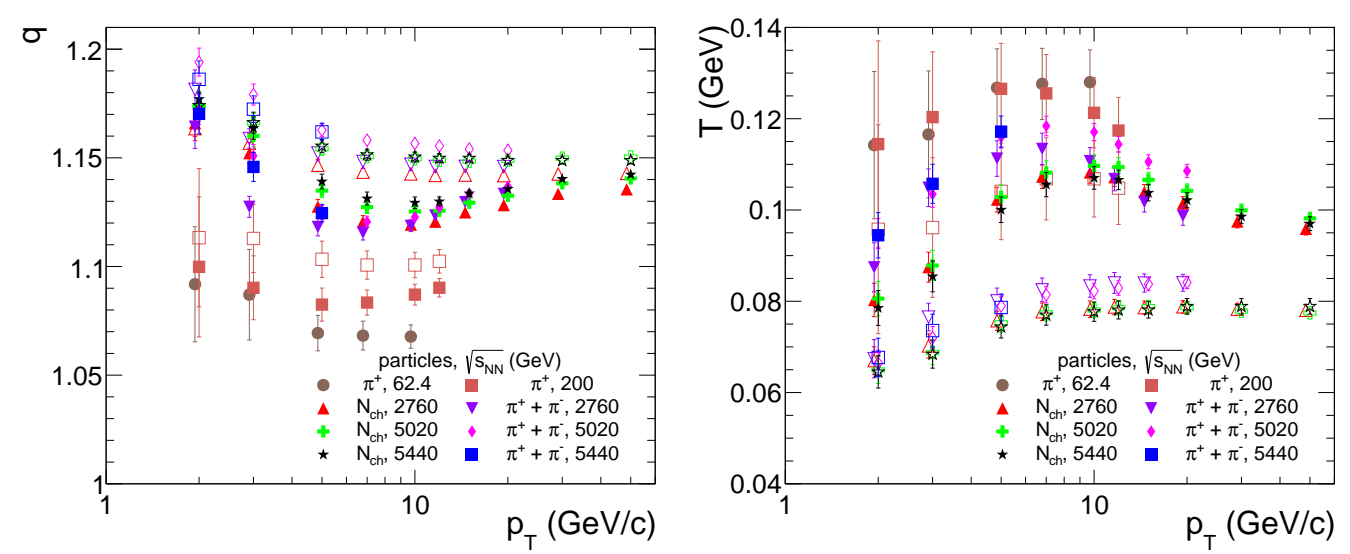

Figure 4: Tsallis parameters $q, T$ as a function $p_{\mathrm{T}}$ of fitting of charged particles $\left(N_{\mathrm{ch}}\right)$ and pions for different $\sqrt{s_{\mathrm{NN}}}$. The open and solid markers correspond to peripheral and central collisions, respectively.

[3] L. Adamczyk et al., (STAR Collaboration), Phys. Rev. C 96, 044904 (2017).

[4] J. Adam et al., (STAR Collaboration), Phys. Rev. C 101, 024905 (2020).

[5] B. Abelev et al., (ALICE Collaboration), Phys. Rev. C 88, 044910 (2013).

[6] S. Acharya et al., (ALICE Collaboration), Phys. Rev. C 101044907 (2020).

[7] S. S. Adler et al., (PHENIX Collaboration), Phys. Rev. C 69, 034909 (2004).

[8] C. Tsallis, J. Stat. Phys. 52, 479 (1988).

[9] C. Tsallis et al., Physica A 261, 534 (1998).

[10] C. Y. Wong et al., Phys. Rev. D 91114027 (2015). Tsallis fit

[11] J.Cleymans and D. Worku, Eur. Phys. J. A 48, 160 (2012).

[12] M. D. Azmi et al., J. Phys. G: Nucl. Part. Phys. 47045001 (2020).

[13] B. Abelev et al., (STAR Collaboration), Phys. Lett. B 655, 104 (2007).

[14] B. Abelev et al., (STAR Collaboration), Phys. Rev. Lett. 97, 152301 (2006).

[15] J. Adam et al., (ALICE Collaboration), Phys. Rev. C 93, 034913 (2016).

[16] B. Abelev et al., (ALICE Collaboration), Phys. Lett. B 720, 52 (2013).

[17] S. Acharya et al., (ALICE Collaboration), J. High Energy Phys. 11013 (2018).

[18] S. Acharya et al., (ALICE Collaboration), Phys. Lett. B 788, 166 (2019).

[19] S. Acharya et al., (ALICE Collaboration), Eur. Phys. J 781, 584 (2021).

[20] Rajendra Nath Patra et al., Eur. Phys. J Plus 136, 702 (2021). 